\title{
Successful ankle replantation in two cases with different presentations
}

\author{
Adzim Poh Yuen Wen ${ }^{1,2,3}$, Mohd Hanifah Jusoh ${ }^{1,4}$, Arman Zaharil Mat Saad ${ }^{1,2}$, \\ Ahmad Sukari Halim ${ }^{1,2}$, Nu'man Wan Ismail Wan Faisham ${ }^{1,4}$, Wan Sulaiman Wan Azman ${ }^{1,2}$ \\ ${ }^{1}$ Hospital Universiti Sains Malaysia, Health Campus Universiti Sains Malaysia, Kota Bharu; ${ }^{2}$ Reconstructive Sciences Unit, School of Medical \\ Sciences, Health Campus Universiti Sains Malaysia, Kota Bharu; ${ }^{3}$ Plastic Surgery Unit, Department of Surgery, Pusat Perubatan Universiti \\ Kebangsaan Malaysia, Kuala Lumpur; ${ }^{4}$ Department of Orthopedic, School of Medical Sciences, Health Campus Universiti Sains Malaysia, \\ Kota Bharu, Malaysia
}

We report our experience of treating two patients with ankle amputation with different presentations. The first case was a clean-cut sharp amputation. The second case was an avulsion injury following a motor vehicle accident in a patient who arrived 8 hours after the injury. Replantation was successful in both cases. In avulsion injuries, a secondary operation for wound coverage is required at a later stage. With good strategy and a support team, encouraging limb survival outcomes are possible post-replantation.

Keywords Amputation / Traumatic / Replantation / Lower extremity

\author{
Correspondence: \\ Arman Zaharil Mat Saad \\ Reconstructive Sciences Unit, School \\ of Medical Sciences, Health Campus, \\ Universiti Sains Malaysia, Kubang \\ Kerian, Kota Bharu 16150, Malaysia \\ Tel: +60-19-335-3038 \\ E-mail:armanzaharil@gmail.com
}

This article was presented at the PRS Korea 2017 on November 10-12 2017, in Seoul, Korea.

Received: March 15, $2018 \bullet$ Revised: January 21, $2019 \bullet$ Accepted: May 30, 2019

pISSN: 2234-6163・ elSSN: 2234-6171・https://doi.org/10.5999/aps.2018.00514・Arch Plast Surg 2020;47:182-186

\section{INTRODUCTION}

Lower leg replantation is controversial, due to discouraging previous experiences and improvements in prosthetic limbs. It was previously assumed that the lack of a sensate plantar surface itself is an unfavourable outcome, and that a replanted foot would therefore be of equal value to a prosthetic limb. Reports of infrapopliteal amputations over the years [1] have shown improved success in revascularization, high rates of plantar sensation recovery (if the tibial nerve is repaired) and encouraging outcomes. We describe two cases lower leg amputation with different presentations, with a focus on the initial management and outcomes. The first case was a clean-cut sharp amputation that was managed by revascularization and ankle fusion, and healed with good function. The second case was an avulsion injury fol- lowing a motor vehicle accident in a patient who arrived 8 hours after the injury, which needed a flap for coverage.

\section{CASES}

\section{Case 1}

A 32-year-old man was assaulted with a sharp sword, resulting in total amputation of the lower extremity at the level of the tibiotalar joint, with clean-cut wound (Fig. 1A). He presented 4 hours after the injury, in a stable hemodynamic condition with no other associated injuries. The patient was immediately transferred to the operating theatre and put under general anaesthesia. Surgery commenced. A two-team approach was taken; one team prepared the stump, and the other, the amputated part. Stabilization of the ankle joint was performed first, using a rush 


\section{Fig. 1. Sword-inflicted amputation of the left ankle}

(A) Preoperative view of amputation at the level of the tibiotalar joint. (B) Immediate postoperative X-rays showing good alignment of the bones with rush rods in situ. (C, D) At 30 days postoperatively, the medial aspect had healed primarily. The lateral aspect had healed secondarily.
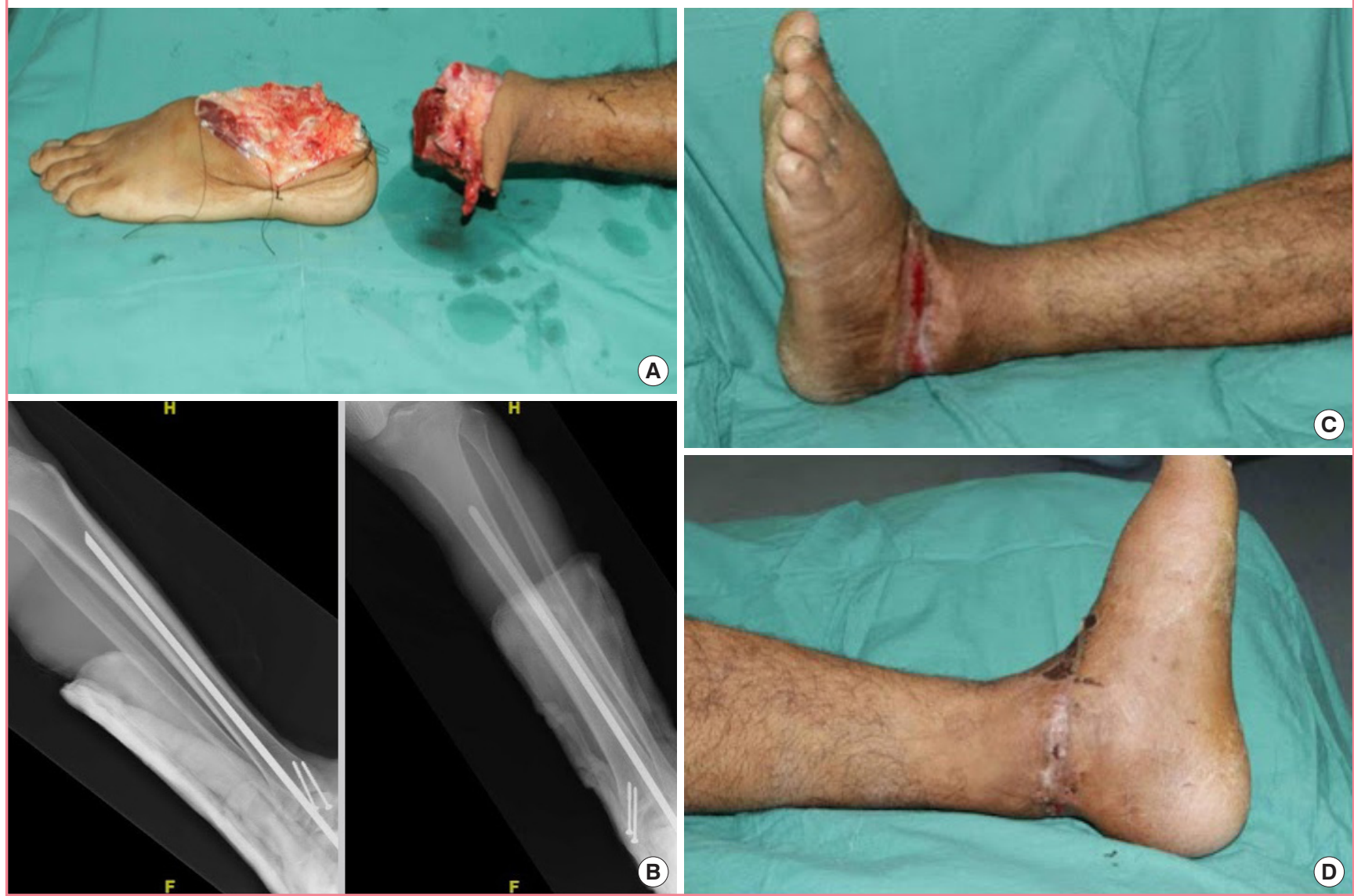

rod inserted from the calcaneum to the tibia bone (Fig. 1B). The blood vessels were then explored, and end-to-end anastomoses were done for the posterior tibial artery, dorsalis pedis artery and its venae comitantes, and the great saphenous vein. Revascularization was completed 6 hours after the injury. The wound edges were approximated, except for the lateral part, which was under some tension and was therefore left to heal secondarily. The patient's intraoperative course was uneventful. He was managed in the intensive care unit for 4 days following replantation. The wound edges healed well, with a dressing only. No complications occurred, and no secondary procedures were needed; as such, the patient showed a good short-term outcome. The replanted foot was viable when the patient was discharged at day 35 postoperatively (Fig. 1C and D). However, this patient was lost to further follow-up, preventing us from assessing his mid- to long-term outcomes.

\section{Case 2}

A 17-year-old boy was involved in a high-impact motor vehicle accident. His right foot was amputated at the level of the tibiota-

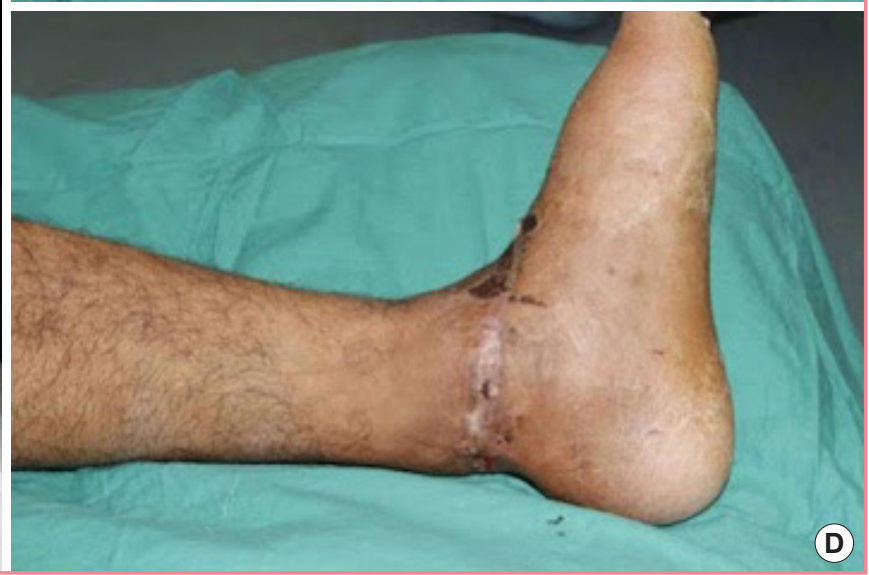

lar joint. The emergency dispatch team wrapped the amputated part of the foot in saline-soaked gauze and kept it in a cooled ice box. A plain radiograph also revealed a distal-third tibia/fibula fracture. The patient was referred to our hospital due to the availability of microsurgery and reconstructive surgery services.

The patient arrived at Hospital Universiti Sains Malaysia 8 hours after the trauma due to the time taken by interstate travel. the time from arrival at the casualty department to the start of surgery was 35 minutes. A similar two-team approach was taken. The wound edge for each respective part was cleaned and debrided. The bone was shortened by a total of $3 \mathrm{~cm}$, and explored for viable vessels. For stabilisation, two rush rods were inserted from the calcaneum to the tibia. Microsurgery commenced, with the posterior tibial artery anastomosed first, followed by its vena comitans. Next, the anterior tibial artery (Fig. 2A and B), with its accompanying vena comitans, and the great saphenous vein were repaired. The tibial nerve (Fig. $2 \mathrm{~A}$ and B) was then coapted. The ischemic time was 10 hours, ending at the time of the first artery repair.

Group repair of the extensor halluces longus and digitorum of 
the tendon was done to maintain clearance of the toes, and the flexor halluces longus and digitorum also were repaired for functional purposes. The skin was then closed with minimal tension. An external fixator was finally applied to reinforce the strength and rigidity of the bone (Fig. 2C).

Ten days postoperatively, the skin edges became necrotic and broke down (Fig. 3A). Wound debridement was carried out, followed by wound coverage with a free anterolateral thigh flap using the vastus lateralis muscle (Fig. 3B). The flap vessels (descending branch of the lateral circumflex femoral artery and vena comitans) were anastomosed to the anterior tibial artery with its venae comitantes via end-to-side anastomosis. The flap healed well, and the patient recovered (Fig. 3C). Three months postoperatively, the patient started partial weight-bearing. A plain radiograph revealed bone union. Six months later, full plantar sensation returned.

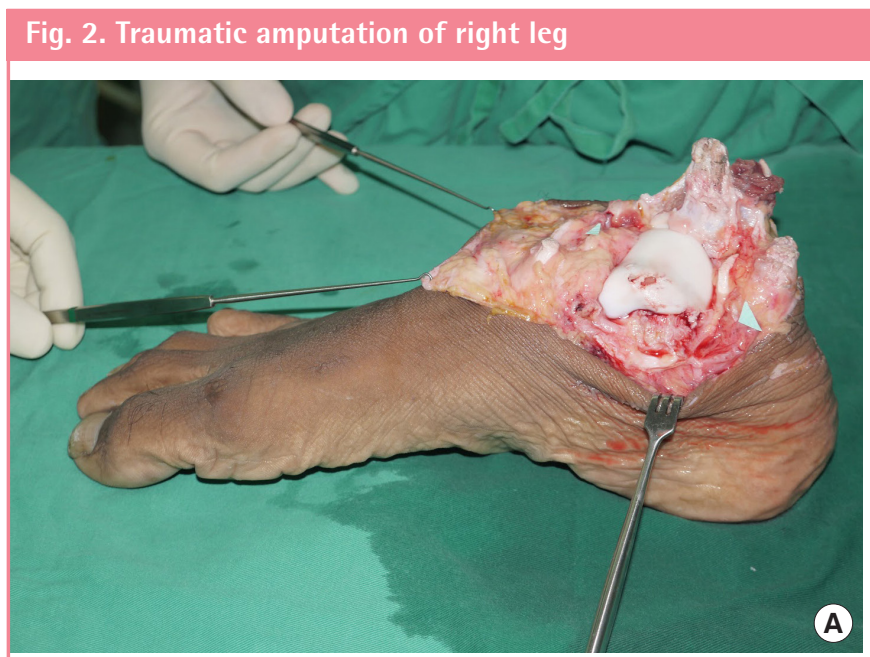

(A, B) Amputation at the level of the tibiotalar joint. Markings showing the tibial nerve and anterior tibial artery. (C) After bone shortening, replantation and microsurgical neurovascular repair were completed. Additional stabilization was performed with an external fixator.
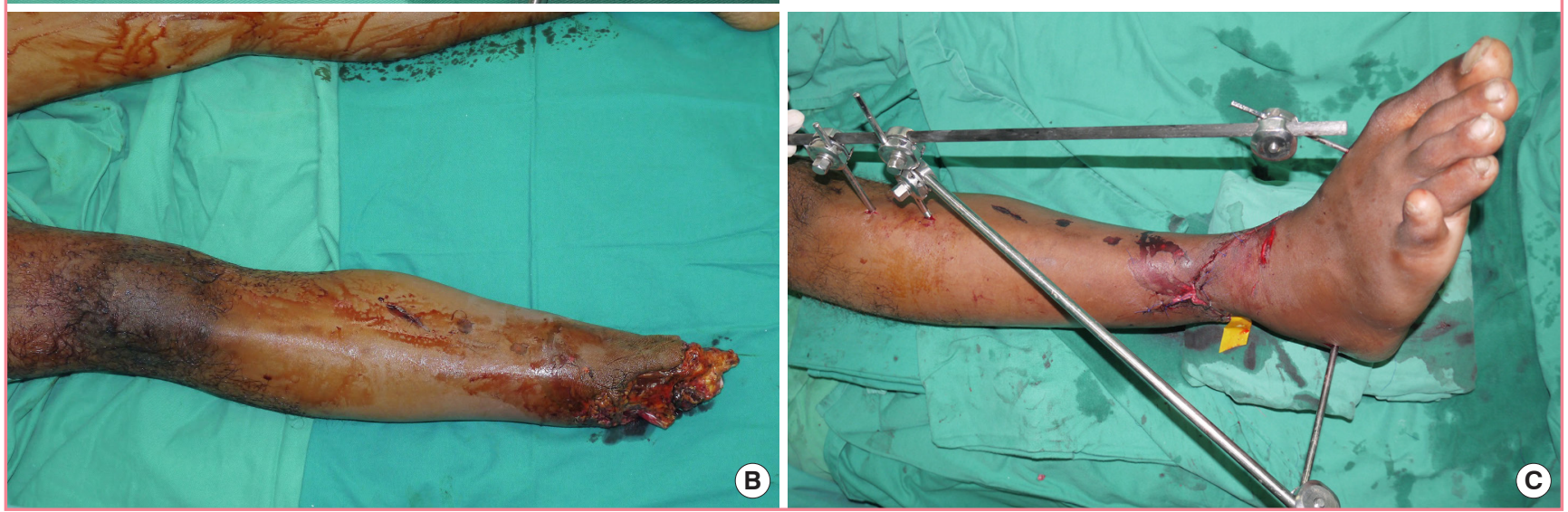

Fig. 3. Wound edge necrosis at 10 days post-replantation

(A) Wound necrosis demarcation. (B) The wound was debrided and covered with a free anterolateral thigh flap. (C) Good viability at 6 weeks after free flap wound coverage.
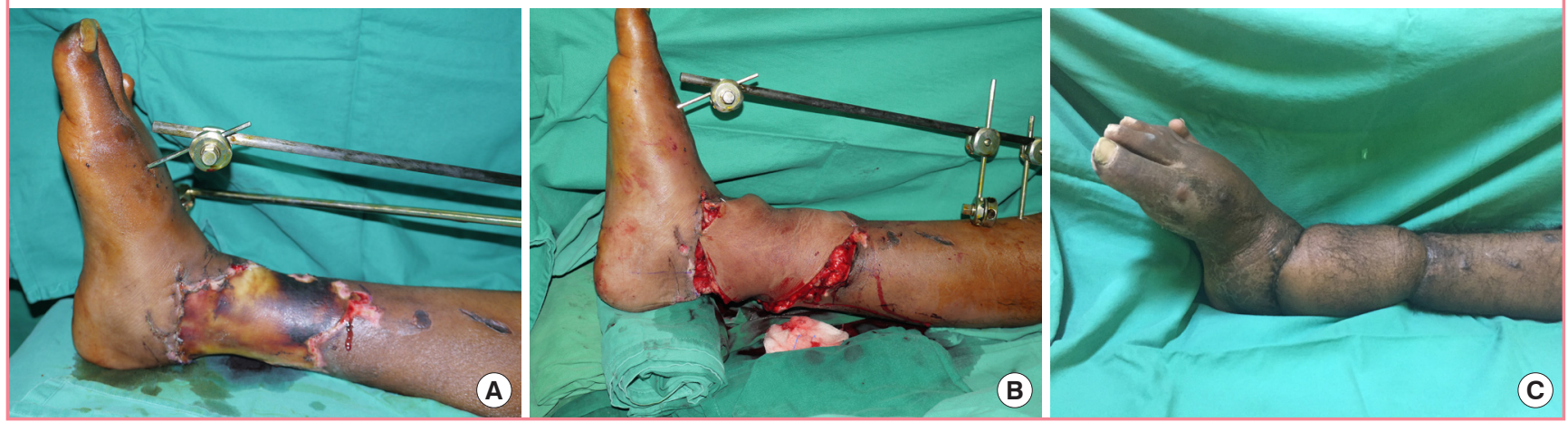


\section{DISCUSSION}

Most reported cases of lower extremity amputation are below the knee level [1]. We have described two cases of ankle/foot amputations, with a focus on differences in presentation and management. Traumatic amputations from road traffic accidents have more damage than clean-cut wounds. The bone edges are less viable, and bone shortening is usually required during debridement, as in our case.

One week post-replantation, we noted wound edge necrosis in the patient who had experienced a road traffic accident. In general, this is expected for avulsion and crush injuries [2], as in the second case. The incidence of soft tissue necrosis and deep infections at the wound edges has been reported to be $83 \%-90 \%$ and $17 \%$, respectively [1]. Nonetheless, rates of bone nonunion or malunion are not different from non-amputation cases [2]. A second operation for wound coverage is common for this kind of injury [2]. In our case, we used a free anterolateral thigh flap with the vastus lateralis muscle. The flap vessels were anastomosed to the anterior tibial artery and its vena comitans in an end-to-side manner, to allow distal foot flow. In contrast, the patient in the first case, in whom the amputation occurred via a cut wound inflicted by a sharp sword, only sustained minimal wound edge damage and did not require any secondary procedures.

Every effort should be made to salvage the amputated part of the limb. In the past, attempts at lower limb replantation resulted in many complications [1]. Noticeable improvements have occurred in the survival rate and outcomes of replanted lower limbs from past to present. This may reflect advances in surgical skills and facilities, resulting in improved ischemic times, as more resources are available in modern healthcare. Likewise, trends have shifted from a tendency to primarily amputate [3] to a more favourable view towards replanting the limb at all costs. In 1983, Kutz et al. [4] reported a 33\% success rate in his case series of nine patients who underwent replantation of the leg. In contrast, in 2002, Battiston et al. [5] reported a $100 \%$ success rate of lower extremity replantation in nine patients, of whom $78 \%$ achieved functional success (Chen grade I or II). In 2005, Hierner et al. [6] reported a $62.5 \%$ success rate in 15 total and subtotal amputated replantations, of which $92.7 \%$ were classified as showing functional success (Chen grade I and II). In a 2009 study by Cavadas et al. [1] in Spain, the survival rate was $100 \%$ in 12 patients with 13 lower leg amputations who underwent replantation.

In the second case (caused by a road traffic accident), the amputated part of the extremity endured 10 hours of ischemic time. Nonetheless, we achieved a good replantation outcome.
Although the generally accepted rule is that the ischemic time should be less than 6 hours [5], Kutz [4] also reported a similar case with ischemic time of 10 hours, in which replantation was successful.

The indications for replantation or amputation of the lower extremity are still not clearly defined. The outcomes predicted by the Limb Salvage Index or Mangled Extremity Severity Score [3] cannot be applied universally to all cases, and there does not seem to be an accurate prediction method $[3,5]$.

It was previously believed that the feasibility of tibial nerve repair to achieve plantar sensation was the most important factor when considering replantation [1]. However, Bosse et al. [3] concluded that, in cases of severe lower extremity trauma, with an insensate plantar surface, the functional outcomes did not differ between salvaged limbs and amputated limbs. In most cases of limb salvage with standard tibial nerve repair, the chances of regaining plantar sensation remain good $[1,4]$. Noticeable improvements have occurred in the survival rate and outcomes of replanted lower limbs from the past to present [1]. In our patient with the sword slash injury, we intended to repair the tibial nerve at a later stage, due to the burdens imposed by microsurgery. However, due to technical and legal issues, the patient was discharged early and did not return for follow-up. A phone conversation with the patient's mother revealed that the patient was walking actively and had resumed daily activities, albeit with a slight limp.

The sequence of vessel repair is controversial. Traditionally, when prolonged ischemia occurs, the artery is repaired first, allowing blood flow to flush out the build-up of toxic metabolites. However, in our case, the foot was deemed to be too small to have accumulated many toxic substances. The sequence of vessel repair also reflects the surgeon's preference, as some surgeons prefer repairing the more difficult vessel while fresh and alert. In other studies, the artery-last sequence has been advocated as a way to promote cleaner and more rapid surgery [2]. Using a two-team approach, as in our cases, also shortens the ischemic time [2]. As a rule, as many vessels as possible should be repaired under microscopy [2].

We used rush rods to achieve both ankle fusion and fracture stabilization. An external fixator was added after wound closure for more structural strength. Perfect rigidity or anatomical correctness of the bone is not as important as vessel repair, and the latter should not be sacrificed for the former [2].

It is advocated to repair at least the tibialis anterior muscle and triceps surae muscle [2]. In our case, it was only possible to repair the tibialis anterior and extensor hallucis longus. However, in a fused joint, as occurred in our case, this step is not the highest priority. The wound should be closed without tension [2], 
with care not to disturb the underlying fragile neurovascular repairs that have just been done.

With improved survival and functional outcomes of replanted limbs, replantation should be a high priority as opposed to primary amputation in cases of total or subtotal lower extremity amputation. All efforts must be directed towards decreasing the ischemic time to achieve the best survival and functional outcomes. No adequate evidence exists for clear guidelines on the decision of whether to salvage a limb.

\section{NOTES}

\section{Conflict of interest}

No potential conflict of interest relevant to this article was reported.

\section{Ethical approval}

The study was performed in accordance with the principles of the Declaration of Helsinki. Written informed consents were obtained.

\section{Patient consent}

The patients provided written informed consent for the publication and the use of their images.

\section{Author contribution}

Conceptualization: Wen APY, Jusoh MH. Writing original draft: Wen APY, Jusoh MH, Wan Faisham NWI. Project administration: Mat Saad AZ, Halim AS, Wan Azman WS. Writing- review \& editing: Wen APY, Mat Saad AZ, Halim AS, Wan Faisham NWI, Wan Azman WS. Approval of final manuscript: all authors.

\section{ORCID}

Adzim Poh Yuen Wen

https://orcid.org/0000-0002-0903-6152

Mohamad Hanifah Jusoh

https://orcid.org/0000-0003-4149-592X

Arman Zaharil Mat Saad

https://orcid.org/0000-0002-4003-6783

Ahmad Sukari Halim

https://orcid.org/0000-0001-8999-6403

Nu'man Wan Ismail Wan Faisham

https://orcid.org/0000-0003-4469-3243

Wan Sulaiman Wan Azman

https://orcid.org/0000-0002-0600-9765

\section{REFERENCES}

1. Cavadas PC, Landin L, Ibanez J, et al. Infrapopliteal lower extremity replantation. Plast Reconstr Surg 2009; 124:532-9.

2. Cavadas PC, Thione A. Lower limb replantation. In: Salyapongse AN, Poore S, Afifi A, et al. editors. Extremity replantation: a comprehensive clinical guide. New York: Springer; 2015. p. 145-59.

3. Bosse MJ, McCarthy ML, Jones AL, et al. The insensate foot following severe lower extremity trauma: an indication for amputation? J Bone Joint Surg Am 2005;87:2601-8.

4. Kutz JE, Jupiter JB, Tsai TM. Lower limb replantation: a report of nine cases. Foot Ankle 1983;3:197-202.

5. Battiston B, Tos P, Pontini I, et al. Lower limb replantations: indications and a new scoring system. Microsurgery 2002; 22:187-92.

6. Hierner R, Betz A, Pohlemann T, et al. Long-term results after lower-leg replantation. Eur J Trauma 2005;31:389-97. 